Pensamiento Crítico No. 7, pág. 97 - 102

\title{
El Cálculo de Probabilidades, la Economía y el Economista
}

Luis Mejía Marcatinco

Palabras claves: Posibilidad, incertidumbre y decisión.

Resumen: El Objetivo es explicar los métodos con los que se puede medir la verosimilitud o posibilidad de sucesos inciertos. En la actualidad, la teoría matemática de la probabilidad es la base para las aplicaciones estadísticas tanto en investigaciones sociales como en la toma de decisiones; para resolver problemas corrientes de la empresa.

De todos las matemáticas que se estudian, ninguna es más importante que el cálculo de probabilidades y sin duda la más utilizada en el campo de la economía.

Cualquiera que sea la profesión que elijan, una cosa es segura: tendrán necesidad de tomar decisiones. Y más de la mitad de las veces habrá de hacerlo en condiciones de incertidumbre y con conocimiento bastante incompleto de las condiciones imperantes. Los directivos empresariales que sean capaces de estimar la evolución de los sucesos futuros tendrán muchas posibilidades de sobrevivir y prosperar en el marco competitivo del mundo de los negocios.

La probabilidad es una parte de nuestras vidas cotidianas. En la toma de decisiones personales y administrativas, nos enfrentamos a la incertidumbre y utilizamos la teoría de la probabilidad, admitamos o no el uso de algo tan complejo. 


\section{Luis Mejía Marcatinco}

Los administradores que se encargan de inventarios de ropa de moda para mujer deben preguntarse las posibilidades de que las ventas alcancen o excedan un cierto nivel. Muchos acontecimientos de nuestra vida carecen de certeza. Están sujetos a la casualidad.

Existen, por ejemplo, la probabilidad de que suene la sirena de los bomberos mientras lees esta frase. También es probable que el profesor dé un escrito hoy. Es difícil predecir exactamente la probabilidad de estos casos nos contentamos con saber que es muy pequeña.

En otros casos, sin embargo, nos gustaría calcular más precisamente la probabilidad, a fin de comparar las posibilidades de diversas alternativas. Pues bien, los matemáticos han estado haciendo precisamente eso hace muchos años, mediante lo que se llama el cálculo de probabilidades.

Actualmente existen más de ochenta diferentes clases de matemáticas. Ningún matemático puede aspirar a conocer más que una parte pequeña de todo esto. En verdad el estudio de cualquiera de estas ochenta diferentes ramas de las matemáticas ocuparían a un genio matemático por toda su vida.

Además, cada día del año se escriben cientos de páginas de nuevas matemáticas, lo que es mucho más de lo que una persona podría leer en ese mismo día. La verdad es que durante los últimos 50 años se han descubierto más matemáticas que en todos los miles de años anteriores de la existencia del hombre.

Una de las ochenta clases de matemáticas que a los matemáticos les resulta interesante y que utilizan para resolver problemas, es el estudio del azar o probabilidad.

\section{Disney necesitó los servicios de los matemáticos}

Antes de que Walt Disney construyera Disneyland, los matemáticos le respondieron a muchas preguntas de esta clase. Cuando se proponía fundar Disneyland, Disney deseaba saber de qué tamaño debía construirla, dónde debería estar situada, cuánto debía cobrar por la entrada y qué clase de entretenimientos debía ofrecer para los días feriados. No deseaba arriesgar una inversión de \$17,000,000 al construir Disneyland sin saber algo de las probabilidades de su buen éxito.

Las preguntas para las cuales Disney deseaba encontrar respuestas eran de este tipo: Si construyo cierta clase de entretenimiento, en determinado lugar y cobró tanto por la entrada, ¿qué probabilidad tendré de obtener cierta ganancia?. 


\section{El cálculo de probabilidades, la economía y el economista}

Disney se fue al Instituto de Investigaciones de Stanford, donde conversó con un grupo de personas especializadas en aplicar el razonamiento matemático a los problemas de los negocios. Lo primero que hicieron los hombres de Stanford fue recoger varias estadísticas acerca de la gente (sus ganancias, sus hábitos de viaje, sus entretenimientos preferidos, número de hijos, etc.). Combinando esta información mediante razonamientos matemáticos, predijeron la probabilidad de que la gente fuera a un lugar dado y pagara cierta suma por derecho de entrada.

Gracias a esta clase de razonamiento, pudieron predecir la probabilidad de tener una Disneyland próspera con cierto tipo de entretenimiento en un determinado lugar. Sabiendo ahora la probabilidad de tener buen éxito bajo ciertas condiciones, Disney estaba mejor capacitado para resolver cómo y dónde construir Disneyland, y cuánto cobrar por la entrada.

Este ejemplo es típico de la manera como se usa la probabilidad para calcular el grado de inseguridad de un acontecimiento o la probabilidad de buen éxito en cualquier acción que se proyecta realizar.

La probabilidad ha llegado a ser un importante campo de estudio con múltiples aplicaciones, que se extiende más allá del dominio de la estadística. Surgen con frecuencia en la toma de decisiones. Y muchas veces necesitamos conocer los métodos con los que se puede medir la verosimilitud o posibilidad de sucesos inciertos para tomar decisiones.

Por ejemplo:

1. Los inversores tienen que decidir si deberá invertir en un valor concreto basándose en sus expectativas sobre rendimiento futuros.

2. Los empresarios, al decidir sobre la comercialización de un nuevo producto, se enfrentan a la incertidumbre de si tendrán éxito.

3. Los directores de establecimientos minoristas han de decidir sobre los niveles de existencias actuales sin conocer las cifras de cuentas futuras, $y$

4. Los grupos de investigación de mercados de las empresas importantes han de emprender planes de publicidad sin el pleno conocimiento de sus efectos.

En estos casos, como ocurre en la mayoría de los asuntos de negocios, se han de tomar decisiones sin disponer de toda la información pertinente.

Todo esfuerzo tendente a disminuir el nivel de incertidumbre en el proceso de toma de decisiones incrementará la posibilidad de tomar decisiones más inteligentes mejor informados. 


\section{Luis Mejía Marcatinco}

Si mejoramos nuestra capacidad de juzgar la probabilidad de ocurrencia de sucesos futuros, podremos minimizar el riesgo y la especulación peligrosa inherente al proceso de toma de decisiones.

En muchas actividades de la industria moderna la probabilidad desempeña un papel muy importante. El control de la calidad y de la confiabilidad de un artículo manufacturado son consideraciones nuevas y muy importantes en las cuales se usa la probabilidad.

Los problemas de confiabilidad pueden resultar muy complejos. Una idea básica de la confiabilidad, sin embargo, puede ilustrarse en la siguiente manera. Se fabrican muchos miles de artículos de cierto tipo; la compañía selecciona al azar 100 muestras de tales artículos y los somete a pruebas cuidadosas. En esas pruebas se halla que 98 de los artículos reúnen los requisitos y se comportan satisfactoriamente. Esto sugiere que 98/100 es una medida de la confiabilidad del artículo. Se puede esperar que alrededor de $98 \%$ de todos los artículos manufacturados por este procedimiento sea satisfactorio. La probabilidad o una medida de la posibilidad de que un artículo fabricado por este procedimiento sea satisfactorio podrían indicarse con 0.98 .

Nos ha tocado vivir en un mundo que cambia muy rápidamente. ¿Te das cuenta de que muchas cosas como la televisión en colores, los submarinos atómicos, los aviones de propulsión a chorro y los satélites artificiales son todas invenciones recientes?. Existen medicinas y vacunas nuevas- También hay diferentes modos de tomar decisiones en los negocios. Hay nuevas maneras de computar. Cada día se hacen cientos de descubrimientos o invenciones.

Lo interesante es que los matemáticos y las matemáticas han contribuido a todas estas formas de progreso.

En la industria telefónica, las matemáticas se usan para diseñar maneras de pasar de un circuito a otro, a fin de que, cuando marcas un número telefónico, tengas buena probabilidad de no encontrarte con una línea ocupada. Las matemáticas han contribuido especialmente al descubrimiento de mejores medios para enviar información a través de hilos telefónicos o mediante sistemas de comunicación inalámbrica.

En la industria de la aviación y en la astronáutica las matemáticas ayudan a determinar la mejor forma para un avión o para una nave interplanetaria, y la necesaria solidez de su construcción. Otra clase de matemáticas predice el riesgo de que un avión se desintegre mientras vuele a grandes velocidades a través de una zona tormentosa. También hay otras formas de matemáticas que ayudan a diseñar la radio y el radar que se usan para guiar los aviones y mediante los cuales unos aparatos pueden comunicarse con otros y con los aeropuertos. 
El cálculo de probabilidades, la economía y el economista

En casi toda clase de fábricas, las matemáticas (el cálculo de probabilidades) se usan para predecir la durabilidad o eficiencia de los objetos fabricados. Muchas veces el fabricante debe garantizar el funcionamiento correcto de su producto basado en una predicción matemática. Si un matemático se equivoca, el fabricante pierde dinero (iy el matemático puede perder su empleo!).

Otras clases de matemáticas nuevas ayudan a los comerciantes a decidir cuánto deben producir, cómo distribuir mejor el tiempo de producción para no tener que pagar sobresueldo a los operarios, y dónde construir nuevas instalaciones a fin de reducir los gastos de transporte.

En la industria petrolera las matemáticas se utilizan extensamente para decidir cuántos pozos deben abrirse, y dónde debe perforarse para obtener la mayor cantidad posible de petróleo con el menor costo. Las técnicas matemáticas también ayudan al fabricante de gasolina a decidir cuántas gasolina de cada clase debe refinar, partiendo de distintos tipos de petróleo crudo.

En todos estos negocios industrias, como asimismo en universidades y organismos gubernamentales, se calcula extensamente mediante las matemáticas y las nuevas computadoras electrónicas.

El razonamiento matemático, y las distintas clases de matemáticas que se han desarrollado, permiten de una manera precisa describir situaciones complicadas y analizar problemas difíciles. El lenguaje de las matemáticas se expresa en símbolos breves, precisos, definidos y usados de acuerdo a reglas lógicas. Esto hace que a menudo sea posible estudiar problemas demasiado complicados como para ser visualizados directamente.

Frecuentemente, el razonamiento matemático predice la posibilidad o imposibilidad de realizar un experimento científico. A veces, la respuesta más útil que un matemático puede encontrar es demostrar sin lugar a dudas que el problema (o la máquina, o el sistema, o el experimento) que se estudia es imposible. EL trabajo matemático puede también mostrar por qué el problema es imposible en la forma actual y sugerir una manera de evitar las dificultades.

Vivimos en un mundo que es incapaz de predecir el futuro con total certidumbre. Nuestra necesidad de tratar con total incertidumbre nos lleva a estudiar y utilizar la teoría de la probabilidad. En muchos casos, nosotros, como economistas preocupados, tendremos algún conocimiento sobre los posibles resultados de una decisión.

Al organizar esta información y considerarla de manera sistemática, seremos capaces de reconocer nuestras suposiciones, comunicar nuestro razonamiento a otras personas y tomar una decisión más sólida que la que tomaríamos sí sólo diéramos palos de ciegos. 


\section{Luis Mejía Marcatinco}

\section{BIBLIOGRAFÍA}

Métodos de Predicación en Economía. Aznar Antonio. Edit. Ariel edición 1993.

Cálculo Alpicado. Hofman Laurece. Edit. Mc Graw-Hill. Edit. 1998.

Estadística Aplicada a los Negocios. Richard L. Levin. Edit. Prentice may, edit. 2001 